OPEN ACCESS

Edited by:

Shintaro Funahashi, Kyoto University, Japan

Reviewed by:

Leonid L. Rubchinsky, Indiana University, Purdue University Indianapolis, United States Shoji Tanaka,

Sophia University, Japan Louis Kang, RIKEN Center for Brain Science (CBS), Japan

*Correspondence: Albert Compte acompte@clinic.cat

Received: 29 May 2021 Accepted: 11 August 2021 Published: 20 September 2021

Citation

Barbosa J, Babushkin V, Temudo A, Sreenivasan KK and

Compte A (2021) Across-Area Synchronization Supports Feature Integration in a Biophysical Network

Model of Working Memory.

Front. Neural Circuits 15:716965. doi: 10.3389/fncir.2021.716965

\section{Across-Area Synchronization Supports Feature Integration in a Biophysical Network Model of Working Memory}

\author{
Joao Barbosa ${ }^{1,2}$, Vahan Babushkin ${ }^{3}$, Ainsley Temudo ${ }^{3}$, Kartik K. Sreenivasan ${ }^{3}$ and \\ Albert Compte ${ }^{1 *}$
}

${ }^{1}$ Institut d'Investigacions Biomèdiques August Pi i Sunyer (IDIBAPS), Barcelona, Spain, ${ }^{2}$ Laboratoire de Neurosciences Cognitives et Computationnelles, INSERM U960, Ecole Normale Supérieure - PSL Research University, Paris, France,

${ }^{3}$ Division of Science and Mathematics, New York University Abu Dhabi, Abu Dhabi, United Arab Emirates

Working memory function is severely limited. One key limitation that constrains the ability to maintain multiple items in working memory simultaneously is so-called swap errors. These errors occur when an inaccurate response is in fact accurate relative to a nontarget stimulus, reflecting the failure to maintain the appropriate association or "binding" between the features that define one object (e.g., color and location). The mechanisms underlying feature binding in working memory remain unknown. Here, we tested the hypothesis that features are bound in memory through synchrony across feature-specific neural assemblies. We built a biophysical neural network model composed of two one-dimensional attractor networks - one for color and one for location - simulating feature storage in different cortical areas. Within each area, gamma oscillations were induced during bump attractor activity through the interplay of fast recurrent excitation and slower feedback inhibition. As a result, different memorized items were held at different phases of the network's oscillation. These two areas were then reciprocally connected via weak cortico-cortical excitation, accomplishing binding between color and location through the synchronization of pairs of bumps across the two areas. Encoding and decoding of color-location associations was accomplished through rate coding, overcoming a long-standing limitation of binding through synchrony. In some simulations, swap errors arose: "color bumps" abruptly changed their phase relationship with "location bumps." This model, which leverages the explanatory power of similar attractor models, specifies a plausible mechanism for feature binding and makes specific predictions about swap errors that are testable at behavioral and neurophysiological levels.

Keywords: working memory, binding, oscillations, multi-area, attractor network

\section{INTRODUCTION}

Working memory, our ability to hold information in mind for short time periods, is a hallmark of cognition but is severely limited on several fronts (Ma et al., 2014). Some of its limitations, such as its capacity, precision, or specific quantitative biases have been successfully accounted for by a family of biophysically-constrained models, mostly on the basis of a ring attractor network that maintains 
memoranda through sustained reverberatory neural activity (activity bumps) (Compte et al., 2000; Edin et al., 2009; Wei et al., 2012; Wimmer et al., 2014; Almeida et al., 2015; Papadimitriou et al., 2015; Nassar et al., 2018; Bouchacourt and Buschman, 2019; Qi et al., 2019; Barbosa et al., 2020). A feature of working memory that constrains the simultaneous storage of several items is the presence of swap errors (Schneegans and Bays, 2019). These errors occur when an inaccurate response to the target item is in fact accurate relative to a non-target item, reflecting the failure to maintain the appropriate association or "binding" between the separate features that define each item (e.g., color and location). The neural mechanisms supporting feature binding remain unclear, with different computational models implementing two alternative hypotheses (Raffone and Wolters, 2001; Swan and Wyble, 2014; Matthey et al., 2015; Schneegans et al., 2016; Pina et al., 2018; Schneegans and Bays, 2019).

The first type of models are based on selective synchronization (Raffone and Wolters, 2001; Pina et al., 2018). In these models, different neuronal populations selective to each feature that define an object are bound together through synchronized oscillatory activity. This would answer the longstanding question of how independently encoded features could be flexibly encoded as a single concept (Singer, 1999). Thanks to this flexibility, at least conceptually, these models do not suffer from combinatorial explosion as an increasing number of feature combinations are considered. There are, however, important questions about the biological plausibility of this hypothesis. Crucially, such a framework would need a temporal encoder that tags bound features by a "temporal code" and a temporal decoder that is able to distinguish which features are associated by detecting ensembles oscillating in precise synchrony. Both the encoder and decoder would thus depend on undefined biological mechanisms for spike coincidence detection (Shadlen and Movshon, 1999), which would struggle with the known high variability of neural spiking in sustained activity (Compte et al., 2003; Shafi et al., 2007). However, there is ample evidence for oscillatory dynamics during working memory. For instance, oscillatory activity in the gamma band (roughly defined between 30 and $100 \mathrm{~Hz}$ ) increases during the mnemonic periods, both locally (Pesaran et al., 2002; Wimmer et al., 2016) and across sites (Lutzenberger et al., 2002; Kaiser et al., 2003; Palva et al., 2011; Kornblith et al., 2016), and further increases with memory load (Howard et al., 2003; van Vugt et al., 2010; Kornblith et al., 2016; Lundqvist et al., 2016). Importantly, gamma-band activity seems to play a functional role, as working memory binding performance is increased when transcranial stimulation at gamma frequency $(40 \mathrm{~Hz})$ is applied at two different sites (left temporal and parietal), but only when in anti-phase (Tseng et al., 2016) in line with monkey electrophysiology showing that different items are stored in different oscillatory phases (Siegel et al., 2009) and the more general framework of phase-coding in working memory (Fell and Axmacher, 2011).

Another class of models achieve feature binding through "conjunction neurons" - neurons that are selective to all features being bound. Since neurons with mixed selectivity are ubiquitous in the brain (Rigotti et al., 2013; Fusi et al., 2016), these models seem more biologically plausible than those relying on unrealistically precise spike synchronization. Nevertheless, they suffer from some important limitations. First, the number of possible combinations explode quickly with an increasing number of features (Matthey et al., 2015; Schneegans et al., 2016; Schneegans and Bays, 2017, 2019; but see Swan and Wyble, 2014). Second, these models do not have independent storage systems for each feature that define an object, to which there is converging evidence (Olson and Jiang, 2002; Wheeler and Treisman, 2002; Xu, 2002; Delvenne and Bruyer, 2004; Bays et al., 2011b; Fougnie and Alvarez, 2011; Parra et al., 2011). See Ma et al. (2014) and Schneegans and Bays (2019) for recent reviews on the experimental evidence that should constrain multi-item working memory models, in particular those aiming to explain feature binding.

Here, we propose a hybrid model that overcomes several limitations from both types of models. We connected two ring attractor networks - one ring representing and memorizing colors and another ring storing locations - via weak excitation. This is an explicit implementation of the independent storage of individual features, where each feature might be represented in different cortical areas (e.g., color in inferior temporal cortex and location in posterior parietal cortex). Within each area, oscillatory mnemonic activity occured naturally through the interplay between fast recurrent excitation and slower inhibitory feedback. Feature binding was accomplished through the selective synchronization of pairs of bumps across the two networks. Furthermore, encoding and decoding of specific colorlocation associations was accomplished through rate coding. Our hybrid model of rate/temporal coding shares the rich explanatory power of classical ring-attractor models of working memory (Edin et al., 2009; Wei et al., 2012; Wimmer et al., 2014; Almeida et al., 2015; Papadimitriou et al., 2015; Nassar et al., 2018; Bouchacourt and Buschman, 2019; Qi et al., 2019; Barbosa et al., 2020) and derives new predictions that can be tested on multiple levels.

\section{MATERIALS AND METHODS}

\section{Neural Network Model}

We extended a previously proposed computational model (Compte et al., 2000). In particular, we connected two onedimensional ring networks via weak, cortico-cortical excitatory synapses governed by AMPAR-dynamics. Each network consists of 2,048 excitatory and 512 inhibitory leaky integrate-andfire neurons fully connected through AMPAR-, NMDAR-, and $\mathrm{GABA}_{\mathrm{A}} \mathrm{R}$-mediated synaptic transmission as in Compte et al. (2000). Moreover, excitatory and inhibitory neurons were spatially distributed on a ring so that nearby neurons encoded nearby spatial locations. All connections were all-to-all and spatially tuned, so that nearby neurons with similar preferred directions had stronger than average connections, while distant neurons had weaker connections. Inhibitory-to-inhibitory and across-network connectivity was untuned. Intrinsic parameters for both cell types and all the connectivity parameters were taken from Compte et al. (2000), except the following for networks 
holding up to two stimuli or capacity-2 networks (notation consistent with Compte et al., 2000):

$$
\begin{aligned}
& G_{E E, A M P A}=0.09 \mathrm{nS}, G_{E I, A M P A}=0.256 \mathrm{nS} \\
& G_{E E, N M D A}=0.24 \mathrm{nS}, G_{E I, N M D A}=0.11 \mathrm{nS} \\
& G_{I I, G A B A}=2 \mathrm{nS}, G_{I E, G A B A}=3 \mathrm{nS} \\
& g_{\text {ext }, I}=2.74 \mathrm{nS}, g_{\text {ext }, E}=3.5 \mathrm{nS} \\
& J_{E E}^{+}=10, \sigma_{E E}=9, J_{E I}^{+}=J_{I E}^{+}=2.4, \sigma_{E I}=\sigma_{I E}=18
\end{aligned}
$$

For networks holding up to three stimuli (capacity-3 networks),

$$
\begin{aligned}
& G_{E E, A M P A}=0.126 \mathrm{nS}, G_{E I, A M P A}=0.256 \mathrm{nS} \\
& G_{E E, N M D A}=0.2 \mathrm{nS}, G_{E I, N M D A}=0.11 \mathrm{nS}, \\
& G_{I I, G A B A}=2 \mathrm{nS}, G_{I E, G A B A}=3 \mathrm{nS} \\
& g_{\text {ext }, I}=2.8 \mathrm{nS}, g_{\text {ext }, E}=3.58 \mathrm{nS} \\
& J_{E E}^{+}=11, \sigma_{E E}=9, J_{E I}^{+}=J_{I E}^{+}=2.6, \sigma_{E I}=\sigma_{I E}=30 .
\end{aligned}
$$

Connectivity across networks was determined by the following conductances (for unconnected simulations, these conductances were set to zero):

$$
\begin{aligned}
& G_{E E, A M P A, \text { across }}=0.45 \mathrm{nS}, G_{E I, A M P A, \text { across }}=0.18 \mathrm{nS}, \\
& G_{E E, N M D A, \text { across }}=G_{E I, N M D A, \text { across }}=0 \mathrm{nS} .
\end{aligned}
$$

These parameters were adjusted to have within-network oscillations, which was accomplished by increasing the ratio between fast and slow excitation, supported, respectively, by AMPAR and NMDAR channels, as previously shown (Compte et al., 2000). The main dynamics described in this study were robust to a broad range of parameter values (Figures 1-4).

\section{Cross-Correlations}

For the cross-correlation analyses, we computed spike counts in bins of $5 \mathrm{~ms}$, collapsing all neurons around the stimulus presentation location (here called a bump, \pm 340 neurons). Moreover, we computed within- and across-network correlations by, respectively, considering neurons in bumps from the same or different circuits. For the cross-frequency correlation plots (e.g., Figure 2B), we further computed the power spectrum of the resulting cross-correlation functions, averaged across all possible (only within- or only across-) pairs of bumps.

\section{Conversion of Spikes Into Local Field Potentials}

For the conversion of simulated spike trains into local field potentials, we convolved the aggregated spike times $\left(t_{s}\right)$ of all the neurons engaged in a bump (or in the network, depending on the analysis) with an alpha-function synaptic kernel:

$$
L F P(t)=\sum_{t_{s}} \Theta\left(t-t_{s}\right) \frac{t-t_{s}}{\tau} \exp \left(-\frac{t-t_{s}}{\tau}\right)
$$

with $\Theta(t)$ being the Heaviside theta function, and $\tau=5 \mathrm{~ms}$.

\section{Phase-Preservation Index}

To measure how an oscillating activity bump kept its oscillatory phase over multiple trials $(k=1, \ldots, N)$ of our simulation, we first converted spike times into local-field potentials (see above). Through wavelet analysis, we determined the phase $\phi^{k}\left(f_{0}, t\right)$ of the LFP at $f_{0}=30 \mathrm{~Hz}$ (the approximate frequency of oscillations in the network) at all time points $t$ of the simulation, and then we used the phase-preservation index (PPI), a method originally developed by Mazaheri and Jensen (2006) for EEG data.

The PPI is defined by taking a reference time point (in our case $t_{\text {ref }}=$ stimulus offset), and then computing the average consistency of the phases at the specific frequency of interest $f_{0}$ with the rest of the time points:

$$
\operatorname{PPI}\left(f_{0}, t\right)=\frac{1}{N}\left|\sum_{k=1}^{N} e^{i \phi^{k}\left(f_{0}, t_{r e f}\right)-i \phi^{k}\left(f_{0}, t\right)}\right|
$$

Phase-preservation index values thus vary between 0 and 1 , with 1 indicating perfect phase consistency.

\section{Extracting Behavioral Output With a Mixture of Gaussians}

The final behavioral output, for simplicity, was extracted by fitting a mixture of two gaussians to the late-delay average activity of the color network. We then selected the central value (color) of the gaussian component with larger amplitude, or stronger mixture component. We fit the mixture of gaussians using the Python function sklearn.mixture.GMM. This algorithmic read-out could be replaced by a biologically plausible downstream network connected to the color circuit, and tuned to be in a winner-takeall regime - i.e., only able to maintain one bump at a time.

\section{RESULTS}

\section{Working Memory Load Modulates Oscillation Power and Frequency}

We built a computational network model of a local neocortical circuit, with excitatory and inhibitory spiking neurons (leaky integrate-and-fire neuron model) connected reciprocally via excitatory AMPAR-mediated and NMDAR-mediated synapses and inhibitory $\mathrm{GABA}_{\mathrm{A}} \mathrm{R}$-mediated synapses (see "Materials and Methods"). The ring-attractor network model was adjusted to support bump attractor dynamics with up to three simultaneous bumps (Edin et al., 2009), and further adjustment of the relative weights of AMPAR- and NMDAR-mediated currents was performed to set active reverberant neurons in the oscillatory regime (Wang, 1999). Using this computational model we started by investigating the dynamics that originated within each network.

In our model, multiple bumps showed anti-correlated oscillatory activity (Figure 1). As we stored more bumps in the network, lateral inhibition originating from simultaneous memories established anti-phase oscillatory dynamics during the memory period. These oscillatory dynamics were irregular, as illustrated in quickly dampened correlation functions (Figure 1A, 


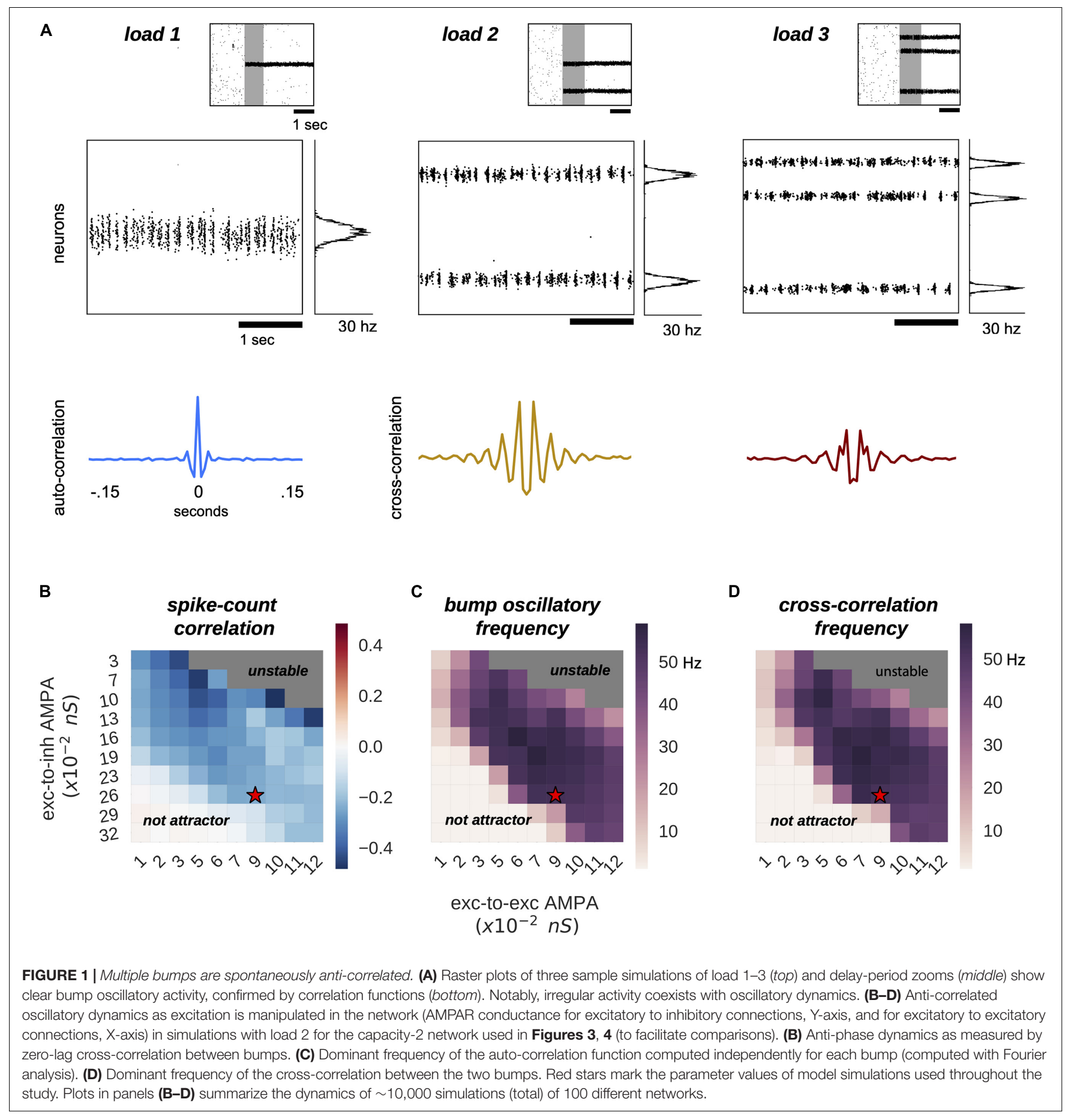

bottom). Moreover, we found that the anti-phase behavior was robust in a wide range of values for AMPAR conductances (Figure 1B), consistently in the gamma range of frequencies (Figures 1C,D). Having seen these anti-phase dynamics between simultaneous bumps, we sought to contrast two opposite scenarios as we increased the number of stored memories (memory load). Under one alternative, bumps may oscillate at a fixed frequency irrespectively of load, so that the global network oscillation (adding up the activity of fixed-frequency out-of-phase bumps) would have a frequency that should increase linearly with memory load (scenario 1, dashed line Figure 2C). Alternatively, the network global oscillation could have a fixed frequency for different loads, and simultaneous bumps would take turns to fire in the available active periods. This would lead to halving each bump's oscillation frequency as we double the memory load (scenario 2, dashed line in Figure 2D). We tested our model simulations to identify if our biophysical model adhered to one of these scenarios. To 
A

\section{network power} peak increases

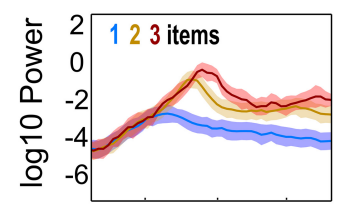

\section{B}

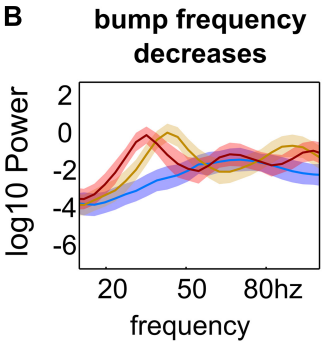

C network power peak increases slightly

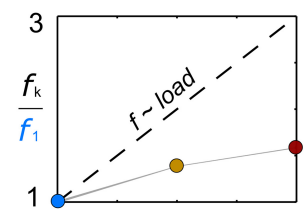

D bump frequency

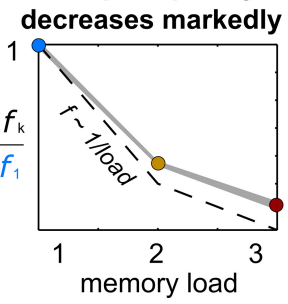

FIGURE 2 | Load-modulation of network and bump oscillatory dynamics. Power spectrum of LFPs computed from simulations with increasing load (1-3), using the activity of the whole network (A) or of each bump's activity (B). (C,D) Peak-frequency $f_{k}$ computed from simulations with increasing load $k$, normalized to frequency $f_{1}$ from simulations with a single bump and computed from LFPs of the whole network activity (C) and from LFPs of each individual bump's activity (D).

this end, we ran multiple simulations with three different loads (presenting 1-3 separate bumps during the encoding cue period) and we computed power spectra from either the aggregate activity of the whole network (network power) or from separate populations centered around each presented target (bump power). We then extracted the frequency of the peak network and bump power to study their dependency with load. We found signatures of both scenarios (Figures 2A,B). As we increased the memory load, the overall network activity oscillated at slightly increasing frequencies (Figures 2A,C). In contrast, each bump, corresponding to different memories, oscillated at markedly slower frequencies as load increased (Figures 2B,D). We quantified which were the dominant dynamics by plotting both the network's and each bump's oscillating frequency against memory load. For better comparison, we normalized the frequency associated with different loads to the one of load 1. Moreover, we compared the effect of memory load against scenario 1 and 2 (dashed lines in Figures 2C,D). Qualitatively, we found that our network dynamics was more consistent with the latter.

We therefore conclude that our biophysical network maintains a relatively constant global oscillation as more items are loaded into memory, and individual memory oscillations instead start skipping cycles to sustain out-of-phase dynamics with other memories. Thus, the interplay between recurrent (fast) excitation and (slower) feedback inhibition acting locally is the basis of the oscillatory bump behavior. Moreover, we now show that anti-phase dynamics of simultaneous bumps occurs due to bump competition, accomplished by lateral inhibition. This competition increases with memory load, leading to longer

periods of silence during the delay-activity of each bump. These dynamics generalize previous findings in simplified rate models (Pina et al., 2018), and extend them to biologically realistic ring attractor networks.

\section{Uniform Coupling Achieves Feature Binding}

The binding between color and location is accomplished through the spontaneous synchronization of pairs of bumps across two networks connected via weak cortico-cortical excitation (Figure 3). In particular, we connected two ring-attractors in the regime described above with all-to-all, untuned excitatory connectivity. This connectivity was weak and it was mediated exclusively by AMPARs (Figure 3A), acting on all excitatory and inhibitory neurons. Interestingly, anti-phase dynamics within each network (as described above) was maintained robustly for a wide range of connectivity strength values (Figures 3E,F). Across networks, each bump's activity was in phase with one bump in the other network (Figures 3B,C, black) but out of phase with the other (Figures 3B,C, red). On the majority of the simulations, this selective synchronization was maintained through the whole delay period (see Figures 3C,D for an example simulation). This set of dynamics is an interesting possible mechanism that binds and maintains the information of each presented stimulus. To this end, however, there are several aspects to resolve in relation to the encoding and decoding of this bound information.

On the one hand, synchronization selection was noiseinduced in our simulations, resulting in across-networks associations between random pairs of bumps for different simulations. To control this association at the time of stimulus encoding, we stimulated strongly ( 7.5 times the intensity of sensory stimuli) and simultaneously one bump in each network for a brief period of $50 \mathrm{~ms}$ (Figures 3B, 4A, green period), forcing these two bumps (one in each network) to engage in correlated activity during the delay period. Nevertheless, this phase-locked dynamics could be broken by noisy fluctuations, leading to possible misbinding of memorized features and swap trials (Figures 4A,B).

On the other hand, our model raised the question of how this binding of information could reasonably be decoded without resorting to complex mechanisms for spike coincidence detection. In our task, the "behavioral" output consisted in answering which "color" was initially associated with a particular "location," and this was accomplished by evaluating which bump of the color network maintained in-phase synchronization with the bump of the probed location at the end of the delay. We found that this did not require complex coincidence detection, but could instead be simulated in a rate formalism as follows. For each trial, we probed one location by stimulating weakly ( $\frac{1}{4}$ of stimulus intensity) corresponding neurons in the location network at the end of the delay. This simulated the visual presentation of a location probe at the end of the delay. This increased the firing rate of the corresponding location bump, and we found that it also resulted in an increase of activity of the associated, in-phase color bump. Finally, we 
A

\section{within-network connectivity}

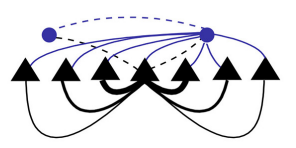

AMPA / NMDA GABAA

B

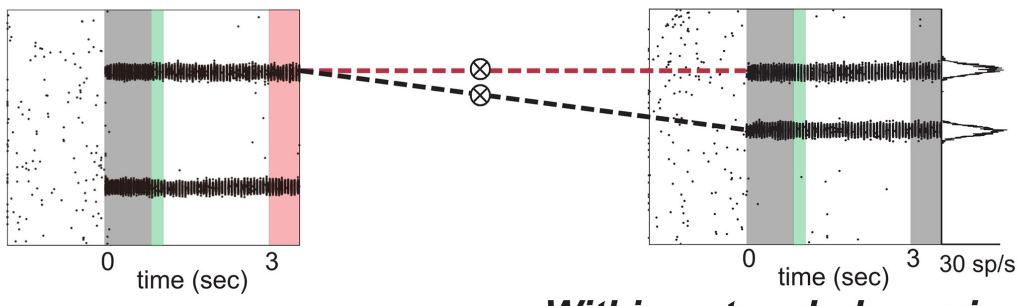

Within network dynamics
E

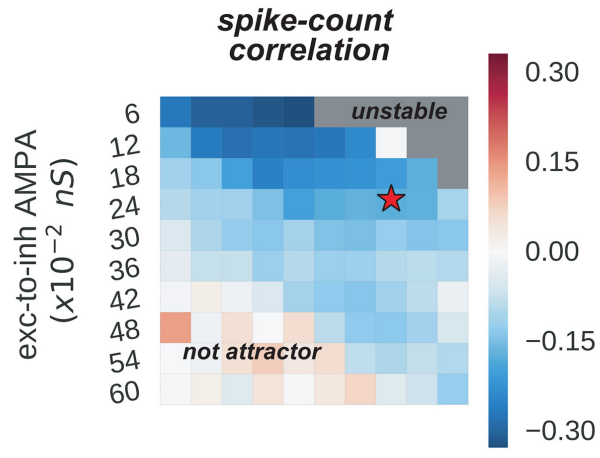

F

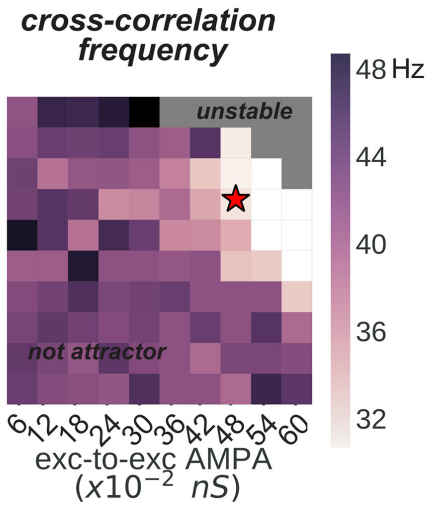

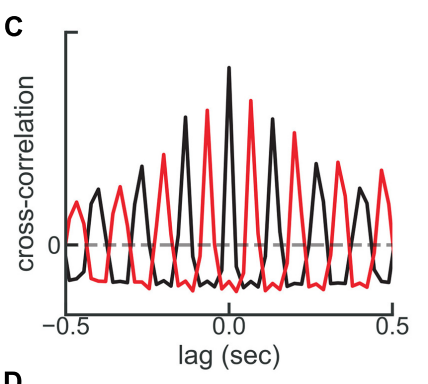

D

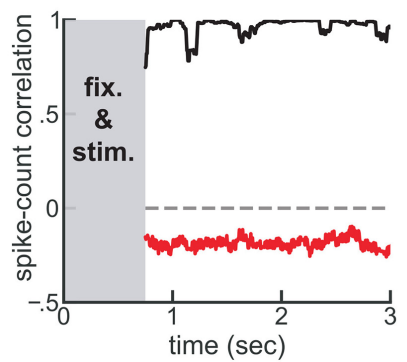

G bump strength
(spike-count std)

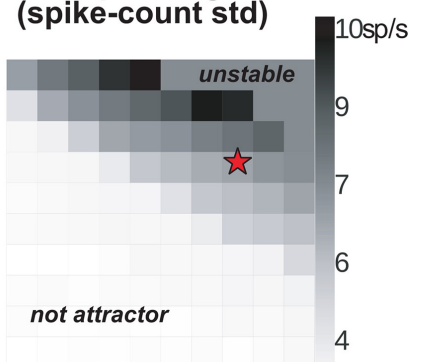

FIGURE 3 | Feature-binding through weak, uniform coupling of 2 ring attractors. (A) Left, schematics of within-network excitatory (black) and inhibitory (blue) connectivity to excitatory (solid) and inhibitory (dashed) neurons. Neurons with similar selectivity were strongly connected as illustrated by the line width. Right, schematics of the 2-network architecture, consisting of 2 ring-attractors with all-to-all, uniform connectivity. Each ring is able to store memories from one feature space (e.g., color or location) as activity bumps (Figure 1). (B) One example simulation for the two networks. The pink-shaded area marks the period in which we read out the activity of the entire color network, while injecting current at one specific location in the location network (right gray-shaded area in the location rastergram, see main text for details about encoding/decoding). (C) Cross-correlation computed between 2 pairs of bumps across networks [as marked with dashed red and black lines in panel (B)]. Across networks, oscillating bumps synchronize in phase (black, positive zero-lag cross-correlation) or out of phase (red, negative zero-lag cross-correlation). (D) Spike count correlation (in count bins of $5 \mathrm{~ms}$ and correlation windows of $100 \mathrm{~ms}$ ) of both associations through the memory delay is stable for this simulation. (E,F) Similar to Figures 1B,D, but manipulating AMPAR conductances across networks. (E) Robustness of anti-phase dynamics within each network as measured by spike count correlation between bumps (Figure 1B). (F) Dominant frequency of cross-correlation between the two bumps within each network (Figure 1D). (G) Bump strength measured as standard deviation of spike-counts across model neurons at the end of the delay. (E-G) summarize the dynamics of 22,000 simulations (total) of $100 \times 2$ networks. Stars indicate parameters and dynamical regime of network simulations shown in panels (B-D).

extracted the behavioral output by fitting a mixture of gaussians ("Materials and Methods") applied to the mean firing rate activity across the color network during the location-probing period (0.5 s). Figure 4B shows color readouts from 1,000 of such simulated trials. Applying our encoding/decoding method to our simulations, resulted in $30 \%$ of trials wrongly associated with the non-target color (swap trials, Figure $\mathbf{4 B}$ ). We then separated swap trials from on-target trials and computed the spike-count correlation in windows of $5 \mathrm{~ms}$ through the whole trial period (Figure 4D), and confirmed that on-target trials were in fact characterized by stable phase-locked activity, while the correlation between bumps in swap trials progressively approached the opposite dynamics (in-phase/anti-phase for the bound/unbound items, Figure 4D). Importantly, networks maintained synchronized in-phase dynamics for bound features robustly over a broad range of inter-network connectivity parameter values (Figures 4E,F). Additionally, we identified three sources of swap errors in our simulations, classified as 
A

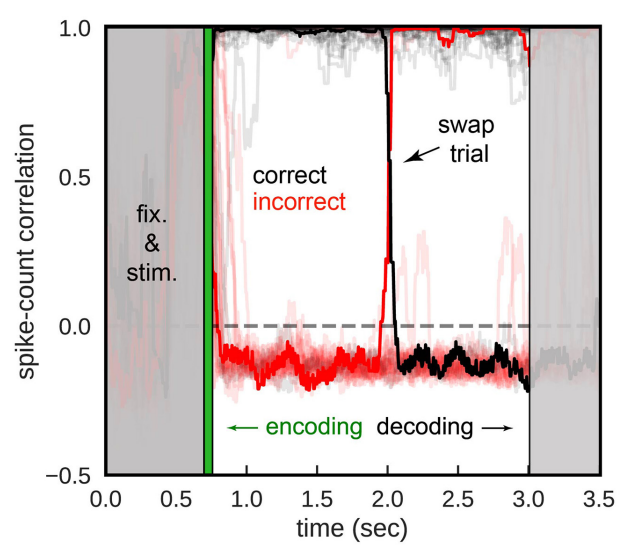

B

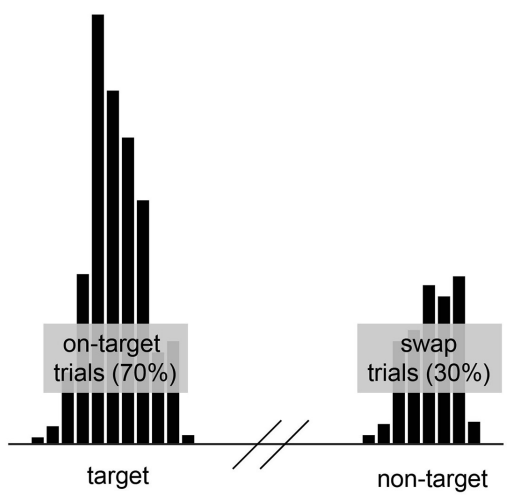

C

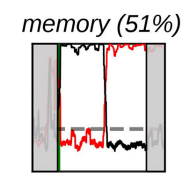

attentional (22\%)

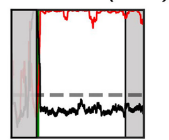

decoding (27\%)

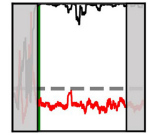

D

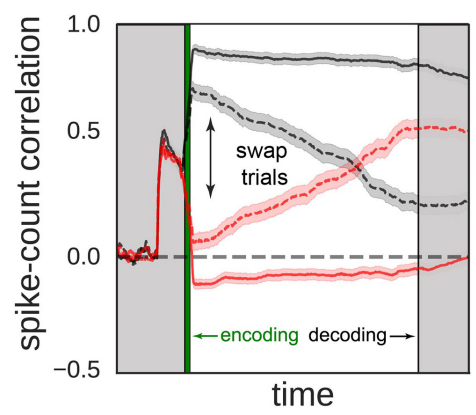

E

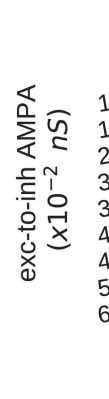

Across networks dynamics

spike-count correlation

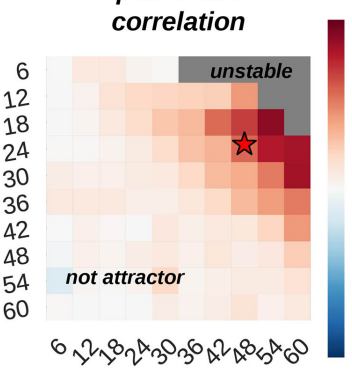

$\mathbf{F}$ cross-correlation 0.8 0.4 0.0 $-0.4$

$-0.8$ exc-to-exc AMPA $\left(x 10^{-2} n S\right)$
G

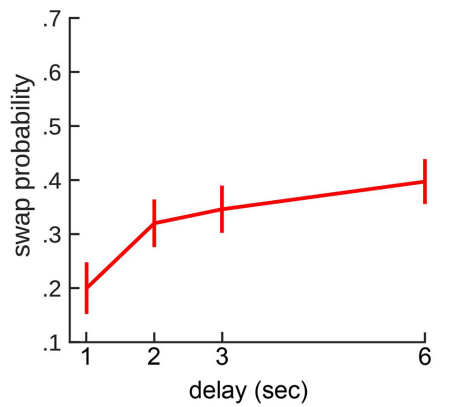

H

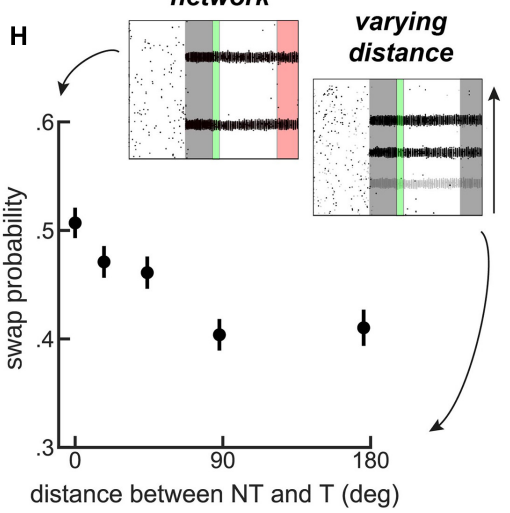

I

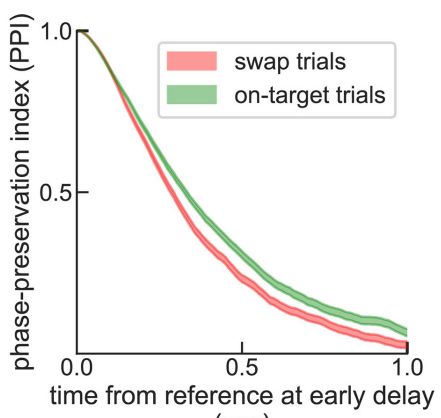

(sec)

FIGURE 4 | Encoding and decoding without temporal precision. (A) Spike-count correlation (in count bins of 5 ms and correlation windows of 100 ms) during the delay for 20 sample simulations. During the encoding period (green), immediately after the stimulus presentation, we bound two bumps, one from each network, by simultaneously stimulating them strongly. This ensured those two bumps were correlated through the trial more often than chance (black lines in the figures), and the other cross-network association synchronized mostly out-of-phase (red lines). On some trials (only one in a), noisy fluctuations reversed these correlations suddenly (swap trials). During the decoding period (light gray, on the right) we simulated the probe period of a working memory task, by stimulating the cued location ( $0.5 \mathrm{~s}$ ) of one network, while decoding mean firing rates from the color network. (B) Color readout histogram in 1,000 simulations. Bumps bound during encoding (target, centered at neuron 520 out of 2,048) were more likely to be read-out than unbound bumps (non-target, centered at 1,480). (C) Three types of swaps: memory swaps (top), attentional swaps (middle), or decoding swaps (bottom). (D) Same as panel (A), averaging across all trials separately for swap and on-target trials, as defined by the decoder, shown in panel (B). (E,F) summary of the dynamics of 22,000 simulations (total) of 100 connected ( $\times 2$ ) networks as a function of inter-network connectivity. (E) Binding stability measured as the average spike-count correlation between initially bound bump pairs during the delay (black, in figures). (F) Dominant frequency of the cross-correlation between bound bump pairs. Red stars mark the parameter values of the model used for sample simulations. (G) Swap errors increase with delay duration and (H) simulations (3 s delay) where target (T) and non-target (NT) bumps are stored close-by (varying 
FIGURE 4 | (Continued)

distance) increase swap error probability, relative to when they are further apart. (I) Swap-error trials (red, $n=3,000$ ), compared with on-target trials (green,

$n=3,000$ ) in the model are associated with a lower phase consistency of oscillatory activity in the delay period, as measured with phase-preservation index (PPI,

"Materials and Methods") using early delay as the reference time point. Error-bars are bootstrapped standard errors $(n=500)$.

memory swaps if the correct association based on in-phase bump synchronization changed abruptly during the delay (51\% of the swap trials), attentional swaps if the wrong association was encoded during the encoding period (22\%) or decoding swaps if the correct association was encoded and maintained during the memory period, but the decoding failed (27\%). See Figure 4C for example simulations.

Together, our biologically-constrained simulations demonstrate that feature-binding can be robustly accomplished through selective synchronization. Crucially, encoding/decoding location-color associations was done without a temporally precise code, a long-standing limitation in the binding by synchrony framework (Shadlen and Movshon, 1999). Moreover, we identified three sources of swap errors. Based on these computational findings, we investigated model predictions that could be compared with existing data or could generate hypotheses for new experimental studies.

\section{Swap Errors Increase With Delay and Item Competition}

In our model, swap errors are induced by noisy fluctuations. This results in two behavioral predictions, congruent with previous findings (Emrich and Ferber, 2012; Pertzov et al., 2017; Schneegans and Bays, 2017). First, longer memory delays should increase the probability of a noisy fluctuation that is sufficiently large to induce a swap (Figure 4G). Second, Figure $\mathbf{4 H}$ shows how swap errors decrease with target to non-target distances. For very close locations, feedback inhibition is strongest, leading to strong competition between nearby bumps, explaining an increase of swap errors for such distances. This is similar to previous studies (Wei et al., 2012; Almeida et al., 2015; Nassar et al., 2018), in which simultaneous bumps interfere (repulsively and through their phase relationship, which is in this case less stable through the delay). Experimentally, these two regimes correspond to different scenarios. In the first case, one color is forgotten, while in the second scenario, there is an actual swap error. This prediction could be tested experimentally by probing the subject's memory on all items, instead of just one (Adam et al., 2017).

In sum, our model is able to describe a previously found dependence of swap errors with delay duration and with target to non-target distance, and it offers mechanistic explanations for such dependencies.

\section{Neural Prediction: Swap Trials Show Less Phase Preservation Through the Delay}

Finally, abrupt changes in the phase relationship between oscillating bumps is the central mechanism of swap errors in our model (Figures 4A,B). Therefore, it is worth deriving a testable neurophysiological prediction from this mechanism. Additionally, because these changes in phase relationships are abrupt, they require experiments using techniques with high temporal resolution such as MEG or EEG. Intuitively, swap errors in our model simulations are characterized by inconsistent phase relationships between brain signals when comparing the beginning and the end of the delay period. We therefore considered applying an analysis that has been proposed to test phase consistency in EEG/MEG: the phase-preservation index (PPI, Mazaheri and Jensen, 2006). We first derived LFP signals from our network's spiking activity ("Materials and Methods"). We then calculated the phase-preservation index (PPI, see Mazaheri and Jensen, 2006 and "Materials and Methods") at the end of the delay, relative to the beginning of the delay, and separately for on-target and swap trials defined "behaviorally" (Figure 4B). As we expected based on our model simulations (Figure 4), this analysis applied to our simulated data showed that trials containing swap errors had a lower PPI, compared to on-target trials (Figure 4I). This prediction can be tested with MEG/EEG data recorded from humans performing this task, based on an analysis of behavioral responses able to discriminate swap and correct error trials (Bays et al., 2009).

\section{DISCUSSION}

Aiming to account for swap-errors, a prominent source of multiitem working memory interference (Schneegans and Bays, 2019), we extended the ring-attractor model (Compte et al., 2000). Our biologically-constrained model offers a plausible mechanism for feature-binding. Briefly, the encoding and decoding of associations is accomplished through rate-coding, while their maintenance is accomplished through selective synchronization of oscillatory mnemonic activity. Oscillatory dynamics emerges naturally from bump competition, which increases with memory load and is in line with previous EEG experiments in humans (Roux et al., 2012) and LFP recordings from monkey PFC (Lundqvist et al., 2018). Finally, our model reveals different origins of swap errors (Mitchell et al., 2018; Pratte, 2019), how they depend on delay duration and inter-item distances (Emrich and Ferber, 2012; Pertzov et al., 2017; Schneegans and Bays, 2017), and predicts that phase-locked oscillatory activity during the memory periods should reflect swap errors.

\section{Other Multi-Area Models for Working Memory}

Our multi-area model adds to a large body of computational work (Ardid et al., 2007, 2010; Edin et al., 2009; Engel and Wang, 2011; Murray et al., 2017; Bouchacourt and Buschman, 2019; Mejias and Wang, 2019; Froudist-Walsh et al., 2020; Min et al., 2020; Novikov et al., 2021) attempting to account for 
the distributed nature of working memory (Christophel et al., 2017). While several of these models have implemented acrossarea interactions through oscillatory dynamics (Ardid et al., 2010; Novikov et al., 2021), they did not attribute a clear mechanistic role to inter-area synchronization dynamics. This is in contrast to our model, where feature-binding in working memory is accomplished through selective synchronization of oscillatory activity in different brain areas.

\section{Comparison With Previous Binding Models}

Previously proposed models by Pina et al. (2018) and Raffone and Wolters (2001) as well as our model are explicit implementations of the synchronization mechanism for feature binding in working memory. While similar in the approach, there are important differences. As argued by Schneegans and Bays (2019), a major difficulty with previous synchronization models was that they were unable to show their capacity of reproducing the rich phenomenology of working memory behavior that other models can explain. Our model, on the basis of its architecture with ring attractor models of spiking neural networks, overcomes the limitation of earlier discrete population models (Raffone and Wolters, 2001; Pina et al., 2018) and keeps all the demonstrated explanatory power that is characteristic of these attractor models, such as explaining several behavioral working memory biases in humans (Almeida et al., 2015; Kiyonaga et al., 2017; Barbosa and Compte, 2018; Kilpatrick, 2018; Nassar et al., 2018; Stein et al., 2020) and monkeys (Papadimitriou et al., 2015; Barbosa et al., 2020); as well as explaining key neurophysiological dynamics during working memory maintenance periods (see Barbosa, 2017 for a short review) in humans (Edin et al., 2009; Kamiński et al., 2017) and monkeys (Wimmer et al., 2014; Sajad et al., 2016). Our model also goes beyond previous synchronization models in that (1) by virtue of its 2 -ring architecture, it explicitly implements the storage of different features in independent systems or brain areas, as shown experimentally (Schneegans and Bays, 2019), and that (2) it provides a plausible rate-based readout mechanism of working memory associations without resorting to complex synchrony detection processes, a major difficulty for this sort of models (Shadlen and Movshon, 1999). Indeed, we show that our proposed mechanisms is robust to the noise inherent in spiking networks, which together with the need of precise spike coincidence detectors were major concerns of the binding through synchronized activity hypothesis in general (Shadlen and Movshon, 1999) and previous implementations in particular (Raffone and Wolters, 2001; Pina et al., 2018).

Thus, our model now brings back synchronization-based feature binding in working memory as a plausible alternative to recent conjunction binding proposals, such as the binding pool (Swan and Wyble, 2014) and the conjunctive coding model (Matthey et al., 2015; Schneegans and Bays, 2017). These models implement binding mechanisms that are fundamentally different from ours. In these models, binding of separated features is accomplished through conjunction neurons, which are neurons selective to mixtures of those features. While there is evidence for such neurons in the cortex (Rigotti et al., 2013; Fusi et al., 2016), their role in feature-binding is not clear, given the consistent evidence for separate feature storage underlying working memory binding (Olson and Jiang, 2002; Wheeler and Treisman, 2002; Xu, 2002; Delvenne and Bruyer, 2004; Bays et al., 2011b; Fougnie and Alvarez, 2011; Parra et al., 2011). Importantly, such a mechanism scales exponentially with the number of feature combinations, thus seemingly inconsistent with our ability to flexibly bind never seen combinations (Schneegans and Bays, 2019). However, it is to be noted that some conjunction models have mitigated this scaling problem through the construction of random conjunctions in an interposed network (Swan and Wyble, 2014; Bouchacourt and Buschman, 2019).

\section{Encoding With Rate Code}

In our hybrid model, only the maintenance of associations is accomplished through correlated oscillatory activity or, in other words, relies on a temporal code. Instead, encoding and decoding of associations is achieved through a rate code. Encoding and decoding is accomplished by delivering flat pulses (i.e., without the need to be temporally precise) to both the to-be-bound features exclusively (encoding) or just to one of them (decoding).

Encoding the association between two different features through a pulse delivered simultaneously to each corresponding bump resembles the sequential encoding hypothesis in working memory (Wolfe, 1994; Bays et al., 2011a). Moreover, there is evidence that a mechanism combining sequential and parallel encoding is implemented in the brain when solving multi-item working memory tasks (Bays et al., 2011a). Our model implements such a combination. First, information about independent features arrives simultaneously to memoryencoding areas from upstream sensory areas. Then, the correct associations are sequentially encoded by brief excitatory pulses, possibly as a result of overt selective attention to each stimulus sequentially (Schoenfeld et al., 2014). Speaking to this, humans take longer to encode combined features than they take to encode the same amount of independent features (Schneegans and Bays, 2019).

\section{Decoding With Rate Code}

Works modelling multi-item working memory though the storage of several bumps in a network (Wei et al., 2012; Krishnan et al., 2018; Nassar et al., 2018) including our own (Edin et al., 2009; Almeida et al., 2015) often used approaches that are biologically implausible to extract the location of one bump, while ignoring other simultaneously maintained bumps. Our approach, however, matches closely the "cueing" period of a multi-item working memory task, which consists of stimulating the "cued" locations while reading out from the whole color network population. Moreover, our encoding/decoding mechanism proposes that swap errors can be of different origins (attention, memory, or decoding; Figure 4C). Indeed, experimental designs that require subjects to rate their confidence on a trial-by-trial basis show that swap errors occur both in highand low-confidence trials, suggesting different origins (Mitchell et al., 2018; Pratte, 2019). 


\section{Future Work: Toward Biological Plausibility of Binding Through Dynamics}

We found anti-phase dynamics within each network and phaselocking across networks, the central mechanisms for featurebinding in our model, to occur naturally in a broad range of parameters, indicating that the mechanisms proposed here do not require fine-tuning. Because our model is to some degree biologically constrained, it is a proof of concept that working memory binding through synchronized activity is at least possible to occur in the brain. In fact, we simulated noisy integrate-and-fire neurons, supporting that the central mechanism implemented in our model has some degree of robustness to noise.

Our model is, however, limited in several ways that could be addressed in future studies. First, we did not simulate trials demanding binding of load 3 or higher. We expect that the main challenges associated with that improvement will be the encoding of more associations. We also did not explore conditions with asymmetric number of bumps (e.g., two colors/locations at/with one location/color), as this would lead to different experimental paradigms. Second, we did not investigate how feature-binding is impacted by incoming distractors. Previous work has shown that oscillatory activity on different bands can play a role in filtering distractors (Dipoppa and Gutkin, 2013). Future work combining these models is necessary. Third, as a proof of concept, we only simulated two connected networks, while humans can encode and decode the association of many more features (Schneegans and Bays, 2019). Relatedly, our two-dimensional network architecture should be taken as a proof of concept, rather than being a literal anatomical representation of a specific brain structure. Finally, the oscillatory regime in which our model is operating, in which neurons are strongly synchronized with the population rhythm (Figure 4C), however, derived from biologically constrained neuronal models, is arguably not biological itself. While there is abundant evidence that neuronal populations show strong oscillatory dynamics in working memory (e.g., Pesaran et al., 2002), single neuron dynamics approaches a Poisson process (Softky and Koch, 1993; Compte et al., 2003) therefore not oscillatory at this

\section{REFERENCES}

Adam, K. C. S., Vogel, E. K., and Awh, E. (2017). Clear evidence for item limits in visual working memory. Cogn. Psychol. 97, 79-97. doi: 10.1016/j.cogpsych. 2017.07.001

Almeida, R., Barbosa, J., and Compte, A. (2015). Neural circuit basis of visuo-spatial working memory precision: a computational and behavioral study. J. Neurophysiol. 114, 1806-1818. doi: 10.1152/jn.00362. 2015

Ardid, S., Wang, X.-J., and Compte, A. (2007). An integrated microcircuit model of attentional processing in the neocortex. J. Neurosci. 27, 8486-8495. doi: 10.1523/JNEUROSCI.1145-07.2007

Ardid, S., Wang, X.-J., Gomez-Cabrero, D., and Compte, A. (2010). Reconciling coherent oscillation with modulation of irregular spiking activity in selective attention: gamma-range synchronization between sensory and executive cortical areas. J. Neurosci. 30, 2856-2870. doi: 10.1523/JNEUROSCI.4222-09. 2010 scale (but see Lundqvist et al., 2016). Early theoretical work (Brunel and Hakim, 1999; Brunel, 2000; Brunel and Wang, 2003) has demonstrated that such oscillatory dynamics at the population level can coexist with noisy, unsynchronized neurons when randomly connected. Future work that connects randomly connected networks that store multiple stable bump-attractors (Hansel and Mato, 2013), but operating in anti-correlated oscillatory activity such as in our simulations could be an appropriate avenue for the future work attempting to overcome these limitations.

\section{DATA AVAILABILITY STATEMENT}

The multi-area model as well as the code for measuring the phasepreservation index is available at https://github.com/comptelab/ binding.

\section{AUTHOR CONTRIBUTIONS}

JB carried out the research. JB and AC conceived the research and wrote the manuscript. KKS, VB, and AT conceived the electrophysiological prediction. All authors edited and approved the final version of the manuscript.

\section{FUNDING}

This work was funded by the Spanish Ministry of Science, Innovation and Universities and European Regional Development Fund (Refs: BFU2015-65315-R and RTI2018094190-B-I00); by the Institute Carlos III, Spain (Grants PIE 16/00014 and AC20/00071); by the Cellex Foundation; by the Generalitat de Catalunya (AGAUR 2014SGR1265 and 2017SGR01565); and by the CERCA Programme/Generalitat de Catalunya. JB was supported by the Spanish Ministry of Economy and Competitiveness (FPI program) and by the Bial Foundation (Ref: 356/18). This work was developed at the building Centro Esther Koplowitz, Barcelona.

Barbosa, J. (2017). Working memories are maintained in a stable code. J. Neurosci. 37, 8309-8311. doi: 10.1523/JNEUROSCI.1547-17.2017

Barbosa, J., and Compte, A. (2018). Build-up of serial dependence in color working memory. BioRxiv [Preprint] doi: 10.1101/503185

Barbosa, J., Stein, H., Martinez, R. L., Galan-Gadea, A., Li, S., Dalmau, J., et al. (2020). Interplay between persistent activity and activity-silent dynamics in the prefrontal cortex underlies serial biases in working memory. Nat. Neurosci. 23, 1016-1024. doi: 10.1038/s41593-020-0644- 4

Bays, P. M., Catalao, R. F. G., and Husain, M. (2009). The precision of visual working memory is set by allocation of a shared resource. J. Vis. 9, 7.1-11. doi: $10.1167 / 9.10 .7$

Bays, P. M., Gorgoraptis, N., Wee, N., Marshall, L., and Husain, M. (2011a). Temporal dynamics of encoding, storage, and reallocation of visual working memory. J. Vis. 11, 6.1-15. doi: 10.1167/11.10.6

Bays, P. M., Wu, E. Y., and Husain, M. (2011b). Storage and binding of object features in visual working memory. Neuropsychologia 49, 1622-1631. doi: 10. 1016/j.neuropsychologia.2010.12.023 
Bouchacourt, F., and Buschman, T. J. (2019). A flexible model of working memory. Neuron 103, 147-160.e8. doi: 10.1016/j.neuron.2019.04.020

Brunel, N. (2000). Dynamics of sparsely connected networks of excitatory and inhibitory spiking neurons. J. Comput. Neurosci. 8, 183-208. doi: 10.1023/a: 1008925309027

Brunel, N., and Hakim, V. (1999). Fast global oscillations in networks of integrateand-fire neurons with low firing rates. Neural Comput. 11, 1621-1671. doi: $10.1162 / 089976699300016179$

Brunel, N., and Wang, X.-J. (2003). What determines the frequency of fast network oscillations with irregular neural discharges? I. Synaptic dynamics and excitation-inhibition balance. J. Neurophysiol. 90, 415-430. doi: 10.1152/ jn.01095.2002

Christophel, T. B., Klink, P. C., Spitzer, B., Roelfsema, P. R., and Haynes, J.-D. (2017). The distributed nature of working memory. Trends Cogn. Sci. (Regul. Ed.) 21, 111-124. doi: 10.1016/j.tics.2016.12.007

Compte, A., Brunel, N., Goldman-Rakic, P. S., and Wang, X. J. (2000). Synaptic mechanisms and network dynamics underlying spatial working memory in a cortical network model. Cereb. Cortex 10, 910-923. doi: 10.1093/cercor/10.9. 910

Compte, A., Constantinidis, C., Tegner, J., Raghavachari, S., Chafee, M. V., Goldman-Rakic, P. S., et al. (2003). Temporally irregular mnemonic persistent activity in prefrontal neurons of monkeys during a delayed response task. J. Neurophysiol. 90, 3441-3454. doi: 10.1152/jn.00949.2002

Delvenne, J., and Bruyer, R. (2004). Does visual short-term memory store bound features? Vis. Cogn. 11, 1-27. doi: 10.1080/13506280344000167

Dipoppa, M., and Gutkin, B. S. (2013). Flexible frequency control of cortical oscillations enables computations required for working memory. Proc. Natl. Acad. Sci. U.S.A. 110, 12828-12833. doi: 10.1073/pnas.130327 0110

Edin, F., Klingberg, T., Johansson, P., McNab, F., Tegnér, J., and Compte, A. (2009). Mechanism for top-down control of working memory capacity. Proc. Natl. Acad. Sci. U.S.A. 106, 6802-6807. doi: 10.1073/pnas.0901894106

Emrich, S. M., and Ferber, S. (2012). Competition increases binding errors in visual working memory. J. Vis. 12:12. doi: 10.1167/12.4.12

Engel, T. A., and Wang, X.-J. (2011). Same or different? A neural circuit mechanism of similarity-based pattern match decision making. J. Neurosci. 31, 6982-6996. doi: 10.1523/JNEUROSCI.6150-10.2011

Fell, J., and Axmacher, N. (2011). The role of phase synchronization in memory processes. Nat. Rev. Neurosci. 12, 105-118. doi: 10.1038/nrn2979

Fougnie, D., and Alvarez, G. A. (2011). Object features fail independently in visual working memory: evidence for a probabilistic feature-store model. J. Vis. 11:3. doi: $10.1167 / 11.12 .3$

Froudist-Walsh, S., Bliss, D. P., Ding, X., Jankovic-Rapan, L., Niu, M., Knoblauch, K., et al. (2020). A dopamine gradient controls access to distributed working memory in monkey cortex. BioRxiv [Preprint] doi: 10.1101/2020.09.07.286500

Fusi, S., Miller, E. K., and Rigotti, M. (2016). Why neurons mix: high dimensionality for higher cognition. Curr. Opin. Neurobiol. 37, 66-74. doi: 10.1016/j.conb.2016.01.010

Hansel, D., and Mato, G. (2013). Short-term plasticity explains irregular persistent activity in working memory tasks. J. Neurosci. 33, 133-149. doi: 10.1523/ JNEUROSCI.3455-12.2013

Howard, M. W., Rizzuto, D. S., Caplan, J. B., Madsen, J. R., Lisman, J., Aschenbrenner-Scheibe, R., et al. (2003). Gamma oscillations correlate with working memory load in humans. Cereb. Cortex 13, 1369-1374. doi: 10.1093/ cercor/bhg084

Kaiser, J., Ripper, B., Birbaumer, N., and Lutzenberger, W. (2003). Dynamics of gamma-band activity in human magnetoencephalogram during auditory pattern working memory. Neuroimage 20, 816-827. doi: 10.1016/S10538119(03)00350-1

Kamiński, J., Sullivan, S., Chung, J. M., Ross, I. B., Mamelak, A. N., and Rutishauser, U. (2017). Persistently active neurons in human medial frontal and medial temporal lobe support working memory. Nat. Neurosci. 20, 590-601. doi: 10. 1038/nn.4509

Kilpatrick, Z. P. (2018). Synaptic mechanisms of interference in working memory. Sci. Rep. 8:7879. doi: 10.1038/s41598-018-25958-9

Kiyonaga, A., Scimeca, J. M., Bliss, D. P., and Whitney, D. (2017). Serial dependence across perception, attention, and memory. Trends Cogn. Sci. (Regul. Ed.) 21, 493-497. doi: 10.1016/j.tics.2017.04.011
Kornblith, S., Buschman, T. J., and Miller, E. K. (2016). Stimulus load and oscillatory activity in higher cortex. Cereb. Cortex 26, 3772-3784. doi: 10.1093/ cercor/bhv182

Krishnan, N., Poll, D. B., and Kilpatrick, Z. P. (2018). Synaptic efficacy shapes resource limitations in working memory. J. Comput. Neurosci. 44, 273-295. doi: 10.1007/s10827-018-0679-7

Lundqvist, M., Herman, P., Warden, M. R., Brincat, S. L., and Miller, E. K. (2018). Gamma and beta bursts during working memory readout suggest roles in its volitional control. Nat. Commun. 9:394. doi: 10.1038/s41467-017-02791-8

Lundqvist, M., Rose, J., Herman, P., Brincat, S. L., Buschman, T. J., and Miller, E. K. (2016). Gamma and beta bursts underlie working memory. Neuron 90, 152-164. doi: 10.1016/j.neuron.2016.02.028

Lutzenberger, W., Ripper, B., Busse, L., Birbaumer, N., and Kaiser, J. (2002). Dynamics of gamma-band activity during an audiospatial working memory task in humans. J. Neurosci. 22, 5630-5638.

Ma, W. J., Husain, M., and Bays, P. M. (2014). Changing concepts of working memory. Nat. Neurosci. 17, 347-356. doi: 10.1038/nn.3655

Matthey, L., Bays, P. M., and Dayan, P. (2015). A probabilistic palimpsest model of visual short-term memory. PLoS Comput. Biol. 11:e1004003. doi: 10.1371/ journal.pcbi.1004003

Mazaheri, A., and Jensen, O. (2006). Posterior alpha activity is not phase-reset by visual stimuli. Proc. Natl. Acad. Sci. U.S.A. 103, 2948-2952. doi: 10.1073/pnas. 0505785103

Mejias, J. F., and Wang, X.-J. (2019). Mechanisms of distributed working memory in a large-scale model of the macaque neocortex. BioRxiv [Preprint]. doi: 10. $1101 / 760231$

Min, B., Bliss, D. P., Sarma, A., Freedman, D. J., and Wang, X.-J. (2020). A neural circuit mechanism of categorical perception: top-down signaling in the primate cortex. BioRxiv [Preprint]. doi: 10.1101/2020.06.15.151506

Mitchell, D. J., Cusack, R., and Cam-Can. (2018). Visual short-term memory through the lifespan: Preserved benefits of context and metacognition. Psychol. Aging 33, 841-854. doi: 10.1037/pag0000265

Murray, J. D., Jaramillo, J., and Wang, X.-J. (2017). Working Memory and Decision-Making in a Frontoparietal Circuit Model. J. Neurosci. 37, 1216712186. doi: 10.1523/JNEUROSCI.0343-17.2017

Nassar, M. R., Helmers, J. C., and Frank, M. J. (2018). Chunking as a rational strategy for lossy data compression in visual working memory. Psychol. Rev. 125, 486-511. doi: 10.1037/rev0000101

Novikov, N., Zakharov, D., Moiseeva, V., and Gutkin, B. (2021). Activity stabilization in a population model of working memory by sinusoidal and noisy inputs. Front. Neural Circuits 15:647944. doi: 10.3389/fncir.2021.647944

Olson, I. R., and Jiang, Y. (2002). Is visual short-term memory object based? Rejection of the "strong-object" hypothesis. Percept. Psychophys. 64, 1055-1067. doi: 10.3758/bf03194756

Palva, S., Kulashekhar, S., Hämäläinen, M., and Palva, J. M. (2011). Localization of cortical phase and amplitude dynamics during visual working memory encoding and retention. J. Neurosci. 31, 5013-5025. doi: 10.1523/JNEUROSCI. 5592-10.2011

Papadimitriou, C., Ferdoash, A., and Snyder, L. H. (2015). Ghosts in the machine: memory interference from the previous trial. J. Neurophysiol. 113, 567-577. doi: $10.1152 /$ jn.00402.2014

Parra, M. A., Cubelli, R., and Della Sala, S. (2011). Lack of color integration in visual short-term memory binding. Mem. Cognit. 39, 1187-1197. doi: 10.3758/ s13421-011-0107-y

Pertzov, Y., Manohar, S., and Husain, M. (2017). Rapid forgetting results from competition over time between items in visual working memory. J. Exp. Psychol. Learn. Mem. Cogn. 43, 528-536. doi: 10.1037/xlm0000328

Pesaran, B., Pezaris, J. S., Sahani, M., Mitra, P. P., and Andersen, R. A. (2002). Temporal structure in neuronal activity during working memory in macaque parietal cortex. Nat. Neurosci. 5, 805-811. doi: 10.1038/nn890

Pina, J. E., Bodner, M., and Ermentrout, B. (2018). Oscillations in working memory and neural binding: A mechanism for multiple memories and their interactions. PLoS Comput. Biol. 14:e1006517. doi: 10.1371/journal.pcbi.1006517

Pratte, M. S. (2019). Swap errors in spatial working memory are guesses. Psychon. Bull. Rev. 26, 958-966. doi: 10.3758/s13423-018-1524-8

Qi, X.-L., Liu, R., Vazdarjanova, A. I., Blake, D. T., and Constantinidis, C. (2019). Nucleus basalis stimulation stabilizes attractor networks and enhances task representation in prefrontal cortex. BioRxiv [Preprint] doi: 10.1101/674465 
Raffone, A., and Wolters, G. (2001). A cortical mechanism for binding in visual working memory. J. Cogn. Neurosci. 13, 766-785. doi: 10.1162/ 08989290152541430

Rigotti, M., Barak, O., Warden, M. R., Wang, X.-J., Daw, N. D., Miller, E. K., et al. (2013). The importance of mixed selectivity in complex cognitive tasks. Nature 497, 585-590. doi: 10.1038/nature12160

Roux, F., Wibral, M., Mohr, H. M., Singer, W., and Uhlhaas, P. J. (2012). Gammaband activity in human prefrontal cortex codes for the number of relevant items maintained in working memory. J. Neurosci. 32, 12411-12420. doi: 10.1523/ JNEUROSCI.0421-12.2012

Sajad, A., Sadeh, M., Yan, X., Wang, H., and Crawford, J. D. (2016). Transition from target to gaze coding in primate frontal eye field during memory delay and memory-motor transformation. Eneuro 3, e0040-16.2016 1-20. doi: 10.1523/ ENEURO.0040-16.2016

Schneegans, S., and Bays, P. M. (2017). Neural architecture for feature binding in visual working memory. J. Neurosci. 37, 3913-3925. doi: 10.1523/JNEUROSCI. 3493-16.2017

Schneegans, S., and Bays, P. M. (2019). New perspectives on binding in visual working memory. Br. J. Psychol. 110, 207-244. doi: 10.1111/bjop.12 345

Schneegans, S., Spencer, J. P., and Schöner, G. (2016). "Integrating "what" and "where": Visual working memory for objects in a scene. Oxford series in developmental cognitive neuroscience," in In Dynamic thinking: A primer on dynamic field theory, eds G. Schöner, J. P. Spencer, and DFT Research Group (Oxford: Oxford University Press), 197-226.

Schoenfeld, M. A., Hopf, J.-M., Merkel, C., Heinze, H.-J., and Hillyard, S. A. (2014). Object-based attention involves the sequential activation of featurespecific cortical modules. Nat. Neurosci. 17, 619-624. doi: 10.1038/nn. 3656

Shadlen, M. N., and Movshon, J. A. (1999). Synchrony unbound: a critical evaluation of the temporal binding hypothesis. Neuron 24, 67-77, 111-125. doi: 10.1016/s0896-6273(00)80822-3

Shafi, M., Zhou, Y., Quintana, J., Chow, C., Fuster, J., and Bodner, M. (2007). Variability in neuronal activity in primate cortex during working memory tasks. Neuroscience 146, 1082-1108. doi: 10.1016/j.neuroscience.2006.12.072

Siegel, M., Warden, M. R., and Miller, E. K. (2009). Phase-dependent neuronal coding of objects in short-term memory. Proc. Natl. Acad. Sci. U.S.A. 106, 21341-21346. doi: 10.1073/pnas.0908193106

Singer, W. (1999). Neuronal synchrony: a versatile code for the definition of relations? Neuron 24, 49-65, 111-125. doi: 10.1016/s0896-6273(00)80 $821-1$

Softky, W. R., and Koch, C. (1993). The highly irregular firing of cortical cells is inconsistent with temporal integration of random EPSPs. J. Neurosci. 13, 334-350. doi: 10.1523/JNEUROSCI.13-01-00334.1993

Stein, H., Barbosa, J., Rosa-Justicia, M., Prades, L., Morató, A., Galan-Gadea, A., et al. (2020). Reduced serial dependence suggests deficits in synaptic potentiation in anti-NMDAR encephalitis and schizophrenia. Nat. Commun. 11:4250. doi: 10.1038/s41467-020-18033-3
Swan, G., and Wyble, B. (2014). The binding pool: a model of shared neural resources for distinct items in visual working memory. Atten. Percept. Psychophys. 76, 2136-2157. doi: 10.3758/s13414-014-0633-3

Tseng, P., Chang, Y.-T., Chang, C.-F., Liang, W.-K., and Juan, C.-H. (2016). The critical role of phase difference in gamma oscillation within the temporoparietal network for binding visual working memory. Sci. Rep. 6:32138. doi: 10.1038/ srep32138

van Vugt, M. K., Schulze-Bonhage, A., Litt, B., Brandt, A., and Kahana, M. J. (2010). Hippocampal gamma oscillations increase with memory load. J. Neurosci. 30, 2694-2699. doi: 10.1523/JNEUROSCI.0567-09.2010

Wang, X. J. (1999). Synaptic basis of cortical persistent activity: the importance of NMDA receptors to working memory. J. Neurosci. 19, 9587-9603.

Wei, Z., Wang, X.-J., and Wang, D.-H. (2012). From distributed resources to limited slots in multiple-item working memory: a spiking network model with normalization. J. Neurosci. 32, 11228-11240. doi: 10.1523/JNEUROSCI.073512.2012

Wheeler, M. E., and Treisman, A. M. (2002). Binding in short-term visual memory. J. Exp. Psychol. Gen. 131, 48-64. doi: 10.1037//0096-3445.131.1.48

Wimmer, K., Nykamp, D. Q., Constantinidis, C., and Compte, A. (2014). Bump attractor dynamics in prefrontal cortex explains behavioral precision in spatial working memory. Nat. Neurosci. 17, 431-439. doi: 10.1038/nn.3645

Wimmer, K., Ramon, M., Pasternak, T., and Compte, A. (2016). Transitions between multiband oscillatory patterns characterize memory-guided perceptual decisions in prefrontal circuits. J. Neurosci. 36, 489-505. doi: 10.1523/ JNEUROSCI.3678-15.2016

Wolfe, J. M. (1994). Guided search 2.0 a revised model of visual search. Psychon. Bull. Rev. 1, 202-238. doi: 10.3758/BF03200774

$\mathrm{Xu}, \mathrm{Y}$. (2002). Limitations of object-based feature encoding in visual short-term memory. J. Exp. Psychol. Hum. Percept. Perform. 28, 458-468. doi: 10.1037/ /0096-1523.28.2.458

Conflict of Interest: The authors declare that the research was conducted in the absence of any commercial or financial relationships that could be construed as a potential conflict of interest.

Publisher's Note: All claims expressed in this article are solely those of the authors and do not necessarily represent those of their affiliated organizations, or those of the publisher, the editors and the reviewers. Any product that may be evaluated in this article, or claim that may be made by its manufacturer, is not guaranteed or endorsed by the publisher.

Copyright (c) 2021 Barbosa, Babushkin, Temudo, Sreenivasan and Compte. This is an open-access article distributed under the terms of the Creative Commons Attribution License (CC BY). The use, distribution or reproduction in other forums is permitted, provided the original author(s) and the copyright owner(s) are credited and that the original publication in this journal is cited, in accordance with accepted academic practice. No use, distribution or reproduction is permitted which does not comply with these terms. 